\section{Into the atom}

\author{
R. H. Dalitz
}

The Atomic Scientists. A Biographical History. By Henry A. Boorse, Lloyd Motz and Jefferson Hane Weaver. Wiley: 1989. Pp. 472. £19.65, \$27.95.

THE development of our knowledge and understanding of the particulate nature of the matter around us is traced by the authors of this book. Boorse, Motz and Weaver describe the field in terms of the lives and personalities of the main contributors to it, and provide substantial accounts of their research achievements.

The book is divided into sixteen chapters, the title of each indicating qualitatively the area of development covered. Each chapter has a number of sections, each with a specific title including the names of the scientists (usually one or two) who, in the authors' views, made the dominant contributions. Some scientists have their name in more than one of these section titles; for example, Rutherford's appears with "The Discovery of the Alpha and Beta Rays from Uranium", "The Transformation of the Elements", "The Alpha Particle and Helium", "The Nuclear Atom" and "Transformation of an Element", whereas Bethe's name appears with the inapt section title "High Energy Physics" in the chapter "Newer Developments in Atomic and Nuclear Theory" and with "Energy Production in Stars". A biographical sketch is given for each scientist named in a section title, often as long as two or three pages, generally in the first section whose title includes his name but sometimes spreading over several.

There are no diagrams nor displayed equations; the text includes a few mathematical expressions and several equations. Boorse, Motz and Weaver are brave men indeed to set out to explain, for example, the quantum mechanics of Dirac, Heisenberg and Schrödinger, or Maxwell's electrodynamics, or nonabelian gauge theories, to non-scientists, under these constraints. They have done very well overall, with some fairly skilful writing. I found only a few paragraphs where I did not at once recognize what was being described. How well a non-scientist would manage is not so clear; the text appears rather forbidding, being relieved only by the breaks between the longish paragraphs and by the small portraits of the scientists named in that section title. In general, the photographs used have not reproduced well, being much too dark; a few have obviously been touched up and at least one shows a distorted, atypical face. Many portraits are missing in the first third of the book, not surprisingly, and the reader feels their absence.
A number of the scientists included are not at all well-known - the names of Herapath, Waterston, Villard and van der Broek appear in section titles, to mention a few - about half of the entries without portraits are for these and other littleknown scientists. The individuals chosen are sometimes surprising: Jensen is cited in "The Nuclear Shell [model]" section title, but not Maria Goeppert-Mayer, who shared their half of the Nobel prize in 1963; E. P. Wigner merits a place in this



Murray Gell-Mann - out in the cold.

company, but his name does not appear in the index, although it is mentioned in the text.

The outstanding omission in the section titles is the name of Murray Gell-Mann, the leading theoretician during all the decades of exploration and comprehension of the strange particles, the K-mesons and the hyperons, and who received the Nobel prize in 1970 for this work. The final section in the concluding chapter, "High Energy Physics", is entitled "Elementary Particles" but bears no name. Surely the name of Gell-Mann would have appeared most natural in that place? He introduced most of the concepts which are now taken for granted in elementary particle physics. Gell-Mann first introduced the classification system we use for these particles, and he first proposed the unitary octet model for the symmetry of their strong interactions. $\mathrm{He}$ put forward the current algebra which links this unitary symmetry with a chiral symmetry. Above all, it is Gell-Mann who was responsible for the successful hypothesis that quarks are the subunits from which the mesons and baryons are constructed.

The last section is the least successful. The authors badly needed to consult an active particle physicist before going into print. As it is, the section gives, perhaps, a fair picture of the situation in the field up to about 1975 , although various models are rather muddled up together. The global symmetry model was never a serious contender and scarcely deserves mention, for example. The authors write as if CP (simultaneous particle - antiparticle and space reflections) symmetry were exact, whereas its violation was established for $\mathrm{K}_{\mathrm{L}}^{0} \rightarrow \pi^{+} \pi^{-}$decay in 1964. They seem to regard SU(6) symmetry as an established symmetry, which it is not. They often write as if all weak interaction processes involve a neutrino, although they do mention some weak non-leptonic decay processes such as $\Lambda \rightarrow p \pi^{-}$. Quantum chromodynamics (QCD) is discussed on the last two pages, but there is an error of notation - the gluon states are like $\mathrm{r} \overline{\mathrm{r}}$, $\mathrm{r} \overline{\mathrm{g}}, \mathrm{r} \overline{\mathrm{b}}, \mathrm{g} \overline{\mathrm{r}}$, and so on, not like $\mathrm{rr}, \mathrm{rg}, \mathrm{rb}, \mathrm{gr}$. The "30-odd arbitrary parameters" of current particle theory are unfairly assigned to QCD, whereas QCD, the theory of the gluon field, involves only one parameter, a scale factor. The quark masses do not stem from QCD, but are generally believed to be due to the electroweak interactions, which violate QCD. The recently discovered weak bosons and their relationship with the electroweak interactions are briefly mentioned on the last page. The second paragraph on that page should have been excised - the idea that free quarks may exist with Planck mass values, their binding to other quarks being due to gravitational forces, has no support whatever.

For an encyclopaedic book, the index provided is inadequate. Many scientists who are mentioned in the text, but not in any section title, are not included, and this lowers the value of the book for the serious reader.

R. H. Dalitz is Professor of Theoretical Physics, Oxford University, Oxford OX1 3NP, UK. 